\title{
RENDRE L'INCROYABLE QUOTIDIEN
}

Fabrication de la vraisemblance dans Plus belle la vie Muriel Mille

\section{La Découverte I Réseaux}

\section{$2011 / 1-n^{\circ} 165$ \\ pages 53 à 81}

\section{ISSN 0751-7971}

Article disponible en ligne à l'adresse:

http://www.cairn.info/revue-reseaux-2011-1-page-53.htm

Pour citer cet article :

Mille Muriel, « Rendre l'incroyable quotidien » Fabrication de la vraisemblance dans Plus belle la vie, Réseaux, 2011/1 n 165, p. 53-81. DOI : 10.3917/res.165.0053

Distribution électronique Cairn.info pour La Découverte.

(c) La Découverte. Tous droits réservés pour tous pays.

La reproduction ou représentation de cet article, notamment par photocopie, n'est autorisée que dans les limites des conditions générales d'utilisation du site ou, le cas échéant, des conditions générales de la licence souscrite par votre établissement. Toute autre reproduction ou représentation, en tout ou partie, sous quelque forme et de quelque manière que ce soit, est interdite sauf accord préalable et écrit de l'éditeur, en dehors des cas prévus par la législation en vigueur en France. II est précisé que son stockage dans une base de données est également interdit. 


\section{RENDRE L'INCROYABLE QUOTIDIEN}

Fabrication de la vraisemblance dans Plus belle la vie

Muriel MILLE 

e feuilleton Plus belle la vie, diffusé du lundi au vendredi sur France 3, est construit sur un paradoxe : défini au départ comme une chronique réaliste de la vie quotidienne d'un quartier populaire de Marseille, le feuilleton s'est rapidement transformé, intégrant des rebondissements et des intrigues criminelles aux péripéties abracadabrantes, et faisant de ce quartier fictif de la métropole phocéenne le théâtre incessant de meurtres, de complots, la proie d'entrepreneurs immobiliers véreux, de mafieux lyonnais, de terroristes tibétains, du diable... Cependant, le feuilleton continue en partie à se présenter comme " réaliste ", " proche des gens ", comme l'indique la présentation de la série sur le site de la maison de production : "Plus belle la vie, véritable feuilleton de proximité ancré dans la société d'aujourd'hui, retrace la vie des habitants du quartier populaire du Mistral, situé au cour de Marseille ${ }^{1}$. » Même si le feuilleton a évolué depuis son concept d'origine, l'ambition de représenter la société contemporaine reste présente, comme l'illustrent les propos du producteur de la série Hubert Besson dans un article de presse consacré au réalisme de la série : «Plus belle la vie est ancré dans notre société, raconte des histoires liées à la société. () Il faut faire une différence entre actualité et réalisme. La réalité de la série est prégnante ${ }^{2}$. " L'adéquation à la réalité reste ainsi une exigence du feuilleton qui entre en contradiction avec la présence d'intrigues multipliant les événements et les rebondissements, et incluant même parfois une dimension fantastique ${ }^{3}$.

La volonté de réalisme constitue ainsi un critère de jugement et une référence tout au long du processus de production, elle se traduit différemment selon les types d'histoires et les moments de la production. C'est cette contradiction que nous voudrions ici explorer en essayant de comprendre comment les agents de

1. Citation extraite du site «plusbellelavie.fr », le site de la maison de production Telfrance dédié à la série. De nombreux sites Internet existent sur la série, un autre site officiel, celui de France 3, et des sites officieux gérés et créés par des fans.

2. Cité dans un article du 7 février 2010 de Pierre Langlais sur slate.fr.

3. Ainsi, depuis le début, des histoires fantastiques ont été mises en scène dans la série, non sans susciter des débats au sein de la production : le personnage de Lucas voyait ainsi des fantômes, le diable est apparu dans la série sous la forme du personnage de Vassago, un homme d'affaires manipulateur, la jeune Sybille a communiqué par télépathie avec son demi-frère Quentin... 
la fabrication de la série mettent au point des mécanismes de contrôle de la vraisemblance malgré la transformation de la série et retraduisent cette attention dans des décisions narratives et techniques. En effet, les fabricants de la série ont un rapport ambigu au réalisme originel de celle-ci, ils reconnaissent volontiers le caractère invraisemblable de la plupart des histoires longues, mais dans le même temps tout le processus de production est travaillé par une attention à la vraisemblance.

Le souci de réalisme se décline en un ensemble de préoccupations différentes selon les étapes de fabrication, et fonctionne ainsi comme une convention de travail au sens d'Howard Becker (Becker, 1988, p. 53) pour les différents fabricants. Ceux-ci en font un critère de décision et d'évaluation du produit fabriqué bien que leurs conceptions du public ou leurs définitions de ce qui est vraisemblable puissent différer en raison de leur trajectoire, de leur expérience sociale et de leur place dans le processus de production (Pasquier, 1995) ${ }^{4}$. Même s'il aurait été fécond de la relier aux différentes expériences de socialisation de ces individus, le choix a été fait de comprendre le fonctionnement de cette attention à la vraisemblance comme préoccupation professionnelle (Becker, 1988, p. 58).

Un des mécanismes de fonctionnement du réalisme en fiction réside dans ce qui a été analysé par Roland Barthes comme " effet de réel », c'est-à-dire «l'objet ni incongru ni significatif » (Barthes, 1968, p. 84) dont la fonction est de « dénoter le réel concret » à travers la référence à un détail concret. Ce sont ainsi en partie ces « effets de réel » qui parviennent à imposer «l'impression de réalité », comme l'explique Jean-Claude Passeron à propos de l'illusion romanesque (Passeron, 1991, p. 214). L'attention au réalisme des fabricants de la série passe ainsi par un travail et une attention à un ensemble d'éléments qui fonctionnent comme " effet de réel », et viennent resituer les rebondissements des intrigues dans un cadre quotidien. Cette exigence déclinée sous forme de préoccupations narratives et techniques est aussi une manière pour ces professionnels de se rapporter au produit qu'ils fabriquent, et par là à leur public (Chalvon-Demersay, 2007b).

La réflexion se fonde sur une enquête en cours constituée par une analyse de la série à partir du visionnage des épisodes et la lecture des résumés, et d'une

4. Ainsi elle explique : «La production des contenus comme le fruit de négociations entre des producteurs issus de milieux sociaux et culturels différents qui sont confrontés à des consommateurs eux-mêmes diversifiés et dont les réactions sont particulièrement difficiles à anticiper 》 (PASQUIER, 1995, p. 6). 
étude du processus de production à partir d'observations des réunions d'écriture, des réunions de préparation du tournage et du tournage lui-même, et à partir d'une série d'entretiens avec les différents participants à ce processus, et en particulier les scénaristes. Nous avons pu recueillir aussi des documents de travail qui permettront ici d'analyser les transformations d'une péripétie, des premières réunions de travail à la diffusion, en particulier à travers les différentes versions des scénarios. Nous avons en effet décidé de nous pencher plus précisément sur les transformations d'une péripétie tout au long du processus de production, ainsi que sur la gestion des conséquences de cette décision scénaristique.

Il s'agit de suivre les dispositifs mis en place pour encadrer et rendre crédible un geste de violence commis par un adolescent dans la série. En suivant les transformations de cet acte particulier, il est possible d'analyser les mécanismes des décisions d'écriture et de réalisation, ainsi que les discussions qu'elles entraînent, pour assumer les conséquences scénaristiques et morales d'un rebondissement en nous intéressant d'abord aux questions de vraisemblance telles qu'elles se posent pour les auteurs dès l'écriture de la série, puis à la manière dont elles se déclinent en interrogations éthiques et en contraintes techniques tout au long du processus de fabrication. La réflexion s'organise ainsi selon trois grandes étapes de la fabrication du feuilleton : l'écriture des histoires, le passage à la version dialoguée, le moment du tournage et de sa préparation. Cela conduit enfin à un retour sur les conceptions du public de la télévision qui se dessinent en creux dans ces interrogations

\section{UNE ATTENTION DIFFÉRENCIÉE À LA VRAISEMBLANCE}

Le lundi 16 mars 2009, la dernière scène de l'épisode 1176 montre le jeune Raphaël Cassagne poignardant l'homme d'affaires Charles Frémont en plein milieu du commissariat du Mistral. Ce sont cette séquence et les discussions qu'elle a suscitées lors de l'écriture et de la réalisation que nous voudrions étudier ici comme point d'appui de l'analyse du rapport des fabricants de la série à la vraisemblance des histoires.

Plus belle la vie, par sa longévité, la quotidienneté de ses épisodes, le caractère infini de sa narration, les multiples niveaux d'intrigue, emprunte des éléments au soap opera tel que le définissent Muriel Cantor et Suzanne Pingree dans un ouvrage dédié à ce type de fiction (Cantor \& Pingree, 1983, p. 22). La série 
s'en distingue en cela que ce ne sont pas les intrigues sentimentales qui constituent le ressort du suspense mais les rebondissements de l'intrigue policière. Chaque épisode se clôt sur une scène finale, le cliffhanger $^{5}$, qui incite, par les interrogations laissées en suspens, le spectateur à revenir le lendemain. Ce procédé est commun autant au roman feuilleton qu'au soap opera, mais dans le cas de Plus belle la vie, cette dernière scène ne porte pas sur les relations sentimentales entre les personnages mais presque toujours sur l'intrigue policière. Par ailleurs, les intrigues se renouvellent beaucoup plus rapidement que dans un " soap ». Cette rapidité conduit les auteurs à chercher indéfiniment de nouvelles histoires et de nouveaux rebondissements pour relancer l'action et le suspense. Cette définition hybride de la série construit aussi les contraintes narratives des scénaristes ainsi qu'un rapport différencié à l'ambition réaliste selon les intrigues.

Avant d'exposer les dilemmes que se posent les scénaristes, il faut décrire l'organisation de l'écriture du feuilleton. Pour écrire 260 épisodes par an, celle-ci s'organise en deux ateliers. Les trames générales des intrigues sont produites tout au long de l'année par un atelier « séquencier » constitué de sept scénaristes et du directeur d'écriture. Toutes les semaines, l'atelier séquencier se réunit et développe en 85 séquences sur la semaine les déroulements des différentes intrigues en cours pour une semaine d'épisodes, diffusés environ trois mois plus tard. Cette première phase d'écriture est collective : les scénaristes discutent des séquences de la semaine et les élaborent par paires avant de les proposer à tout le groupe. Un second atelier, l'atelier dialogue constitué de sept personnes, un auteur par épisode et de deux responsables, prend ensuite en charge l'écriture des versions dialoguées des scénarios. Le texte est lu, relu, raconté, discuté tout au long de sa fabrication. Cette circulation du texte est bien décrite par Sabine Chalvon-Demersay et Dominique Pasquier dans un article sur la naissance d'un feuilleton: "une véritable navette s'établit et les textes, comme des textes de loi entre les assemblées, circulent d'un groupe de lecture à un autre, au cours d'examens successifs durant lesquels ils sont corrigés, amendés, surchargés » (Chalvon-Demersay \& Pasquier, 1991, pp. 105106). Ici, la navette des textes est extrêmement rapide et donne lieu à chaque étape de la fabrication à des discussions collectives.

5. Le cliffhanger est un terme provenant des serials, films en série américains qui s'achevaient sur des scènes au suspense haletant pour amener le spectateur à venir voir la suite au cinéma, avec l'exemple emblématique du héros suspendu dans le vide, accroché au bord d'une falaise. 


\section{Une attention aux conséquences plus qu'à la péripétie : un traitement différencié des intrigues}

Cette scène constitue donc un cliffhanger puisqu'elle laisse les spectateurs dans le doute sur l'issue du geste de Raphaël : Frémont est-il mort ? L'arche narrative principale raconte l'enquête des policiers du quartier du Mistral sur un trafic de clandestins dirigé par un mystérieux Kamsky ; ils soupçonnent l'ancien avocat et homme d'affaires Charles Frémont, " méchant 》 récurrent de la série, d'être ce Kamsky. Dans le même temps, Jennifer, une jeune fille au passé de délinquante, devient la petite amie de Raphaël, lycéen du Mistral. Elle continue à être mêlée à des trafics louches, et semble en lien avec Kamsky, celui-ci lui ordonnant de retrouver une jeune clandestine qui s'est échappée d'un convoi. Elle tarde à le faire et, le vendredi 13 mars, la dernière scène montre la découverte du corps de Jennifer dans le coffre d'une voiture. L'homme d'affaires Charles Frémont qui servait d'intermédiaire entre Kamsky et la jeune fille est rapidement soupçonné d'être à l'origine de cet assassinat. Il est arrêté dans la journée du lundi. Le lundi soir, en plein commissariat, Raphaël poignarde Frémont qu'il rend responsable de la mort de son amie.

L'arche narrative dans son ensemble porte sur la recherche de l'identité de ce Kamsky, et sur la présence d'agents corrompus au sein de la police. Ce rebondissement n'est pas essentiel pour l'histoire, mais il sert ici à alimenter la recherche perpétuelle de suspense, il place dès le départ les auteurs face à une contradiction née des conséquences de ce geste : Frémont s'en sort indemne, mais Raphaël a commis un geste grave. Or Raphaël est un personnage récurrent, il doit continuer à apparaître à l'écran, la série ne peut donc raconter les conséquences probables de son geste, qui devraient l'éloigner pour un temps. Cette tentative de meurtre se répercute dans la suite de l'intrigue principale. La narration est alors construite autour du séjour de Frémont à l'hôpital, mais elle a un effet incontrôlé qui est discuté tout au long de la fabrication : elle laisse cet acte grave sans conséquences. Cela pose un problème de vraisemblance aux auteurs qui, dès les premières étapes de l'écriture, sont placés ici devant les contradictions de la série.

L'équipe d'auteurs du séquencier, comme le reste des acteurs du processus de production, ne se pose pas le problème de la vraisemblance à propos de la péripétie elle-même. La tentative de meurtre en plein commissariat n'est pas vraiment discutée, les arches A (histoires principales de la série) sont d'ailleurs construites sur des rebondissements spectaculaires et des actes qui sortent de 
la réalité quotidienne : enlèvement, prise d'otage, tentative de meurtre, meurtres, arrestations se succèdent. Cette accumulation les conduit parfois à s'étonner de ce que les spectateurs puissent considérer la série comme réaliste :

Souvent, les gens me disaient quand je leur disais que je travaillais pour Plus belle la vie, genre la caissière, ils me disaient : « ouais, mais c'est comme dans la vie ». Alors que genre, dans la vie, en un an, il vous arrive ce qui arrive à un personnage de Plus belle la vie, mais vous faites une déprime. (Dialoguiste)

Malgré tout, lors de l'écriture et tout au long du processus de production, la question de l'adéquation du feuilleton à la réalité sociale française contemporaine se pose pour les fabricants de la série, sous l'angle ici des conséquences de ce geste et non du geste lui-même. La question est posée dès la première réunion de l'atelier séquencier. Les auteurs des « histoires » se retrouvent en effet deux après-midi et une matinée par semaine pour discuter et inventer ensemble le développement en séquences des arches narratives. Ils travaillent par paires sur une partie des intrigues en cours puis soumettent au groupe, et plus particulièrement au directeur d'écriture, les résultats de leur travail. Lors de la remise en commun, les grandes étapes de l'arche A sont donc évoquées, et en particulier le geste de cet adolescent qui suscite tout de suite des questions :

Séquenceur $1^{6}$ : et qu'est-ce qu'il lui arrive à Raphaël à l'heure où les enfants de 12 ans vont en prison?

Séquenceur 2 : ça serait une bonne $\mathrm{B}$ à faire, sur les mineurs en prison

On voit ici un des principes de construction narrative de la série qui va déterminer la manière dont seront traitées les conséquences de l'acte de Raphaël : en tant que péripétie de "l'intrigue A » (ou arche A), les questions en jeu dans le traitement de la délinquance des mineurs ne sont pas vraiment abordées, ce type de question est plutôt du ressort des intrigues de « type B », qui traitent de l'actualité sociale, de ce qui est appelé " problèmes de société ». Cette coexistence de deux types d'intrigues a constitué un moyen de concilier la définition initiale de la série avec les transformations ultérieures du type de narration. Pour les d'intrigues $\mathrm{B}$, l'attention des auteurs à la vraisemblance est encore plus forte, puisque celles-ci ont souvent une ambition pédagogique.

6. Les noms des enquêtés ont été remplacés par leur fonction dans le processus de production Les dialoguistes et séquenceurs sont numérotés selon leur ordre d'apparition dans les extraits. Les extraits d'observation cités dans ce texte ont été réalisés de novembre 2008 à février 2009. 
Cela crée un rapport différencié à la réalité sociale et à la mission de la série selon les intrigues : traiter de la situation des mineurs en prison rentrerait dans le cadre des " arches » à vocation sociale et pédagogique, mais pas dans le cadre de " l'arche A ». Ici, malgré le fait que l'on se situe dans le cadre de l'intrigue policière, définie par les auteurs eux-mêmes comme irréaliste, il est intéressant de voir que le problème de l'adéquation à la réalité est soulevé par ceux-ci. Ainsi, une fois que s'est posé pour les auteurs le problème du réalisme de l'absence de conséquences de cette action, une intrigue B sur les suites judiciaires de l'acte de Raphaël est créée. Cela fait l'objet d'un développement de l'intrigue la semaine suivante, non prévu au départ. Ce développement met en scène le père du jeune homme discutant avec un juge des suites juridiques de l'acte de son fils, puis demandant à la victime Frémont de retirer sa plainte, et enfin le jeune homme vient s'excuser auprès de celui-ci. Dans ces confrontations, la souffrance psychique de l'adolescent est mise en avant. La séquence d'excuse met ainsi face à face agresseur et victime en un retournement de situation, puisque c'est Frémont qui en vient à présenter ses excuses et ses condoléances pour la mort de la jeune fille. Cette scène remet les choses dans l'ordre : dans la série, le méchant c'est Frémont, ce n'est pas Raphaël, dont le geste est relativisé. La dernière scène montre Benoît insistant sur le fait que la procédure n'est pas finie, et sur la préparation du dossier avec l'avocat. On finit sur le mal-être de Raphaël, et les épisodes suivants ne mentionnent plus ce geste ni ses conséquences. Au bout de quelques épisodes, la succession des intrigues, et le tourbillon narratif de la série font que cette histoire n'est plus mentionnée et que le jeune homme oublie assez rapidement son deuil. Les conséquences juridiques ont été traitées tout en proposant une justification du geste du jeune homme sous la forme d'une intrigue secondaire qui ne dure qu'une semaine. C'est ici un autre aspect de ce rapport différencié à la vraisemblance : selon les intrigues, les personnages évoluent davantage à travers les intrigues de type $\mathrm{B}$, c'est-à-dire considérées comme plus « réalistes » que par celles de type A, c'est-à-dire davantage aussi par celles concernant les relations familiales et sentimentales entre les personnages que par leurs aventures policières. Les personnages changent peu, mais ils sont marqués par les « arches B », alors que les « $\mathrm{A}$ » les laissent souvent inchangés. Le personnage de Raphaël est ainsi défini par sa relation à sa sœur et à son père, par le divorce de ses parents, et il ne change pas suite à cette tentative de meurtre, et ne porte pas longtemps le deuil de son amie. Un des mécanismes de gestion de la contradiction de la série est ainsi l'accumulation des péripéties et des histoires, la série ne pouvant rappeler à chaque instant ce qui s'est passé auparavant. À la mémoire sélective des personnages correspond 
l'oubli progressif des événements sous l'abondance des rebondissements par les spectateurs. Cet oubli se retrouve parfois chez les auteurs eux-mêmes qui oublient les intrigues passées au fur et à mesure qu'ils se consacrent à l'écriture de nouveaux épisodes. À la nécessité d'oublier ce qu'ils ont fait pour rester créatifs correspond l'oubli supposé de téléspectateurs qui doivent être sans cesse surpris.

\section{Le jeu de « l'effet de réel »}

Le point de départ des discussions et des interrogations des auteurs est bien que la société de Plus belle la vie est censée être le plus proche possible de la société actuelle en comparant les deux mondes, comme le montrent la question du séquenceur 1 citée plus haut ou encore les réactions des scénaristes par la suite. C'est en particulier du point de vue juridique que les conséquences de cette action apparaissent problématiques pour les auteurs. Lors de la réunion du lendemain ${ }^{7}$, ils discutent de la scène débutant le lundi matin où Benoît, le père de Raphaël vient chercher son fils au commissariat :

Séquenceur 1 : normalement, c'est direct en taule, et c'est 5 ans.

Séquenceur $3:$ c'est pas vraisemblable.

Directeur d'écriture : le juge peut décider de le remettre en liberté.

Séquenceur $2:$ c'est juste pas réaliste.

Séquenceur 1 : faut l'assumer.

Séquenceur 3 (vérifie sur un des ordinateurs placés dans la salle de réunion, et regarde sur Internet) : le tuteur légal doit être là.

Ce qui est en jeu ici, c'est bien de faire correspondre le sens de ce qui se passerait probablement dans la vie avec ce qui se produit dans la série. La mention puis la mise en scène des conséquences juridiques semble presque fonctionner comme un effet de réel, elle n'a pas réellement de fonction narrative. Dès la discussion et l'élaboration, les auteurs jouent avec le cadre légal pour faire rentrer tant bien que mal l'intrigue dans celui-ci. Alors même que de nombreux événements extraordinaires ont eu lieu, la plupart des intrigues se déroulent en mettant en scène la procédure judiciaire française, et en donnant des dénominations juridiques aux différents crimes ou actions commises.

7. L'atelier séquencier se réunit le jeudi après-midi, le vendredi après-midi et le lundi matin pour développer en groupes les intrigues en séquences. 


\section{Un réalisme des émotions}

Parallèlement, un dispositif scénaristique est mis en place pour donner une justification à l'acte de Raphaël. Les différentes séquences mettent donc en scène son deuil, en montrant Raphaël dans sa chambre successivement consolé par son ami Nathan, sa sœur, son père... ou encore revenant de l'enterrement de Jennifer. Les séquences insistent sur le chagrin du jeune homme qui devient la justification de son geste. En effet, dans les discussions des auteurs, la cohérence des intrigues passe le plus souvent par la justification psychologique des actes, l'acte peut être peu vraisemblable en général, mais il est motivé par les sentiments et l'émotion ressentis par le jeune lycéen. L'explication souvent donnée par les scénaristes face aux réflexions sur l'irréalisme de la série est que même si les intrigues peuvent paraître invraisemblables, le principal est que les réactions des personnages soient justes. Lors des discussions de l'atelier séquencier, les scènes sur le chagrin de Raphaël sont décrites ainsi : «on a repsychologisé l'axe Nathan-Raphaël », commente le directeur d'écriture en présentant aux autres auteurs leur travail de l'après-midi. Le réalisme est alors placé dans la justesse des sentiments exprimés par les personnages, comme l'explique le responsable de l'atelier dialogue :

C'est-à-dire que ce qui est important dans Plus belle la vie, ce sont les sentiments. En fait, ce sont les personnages et ce que vivent les personnages. Et ce qu'ils vivent à travers leurs histoires, quoi. Et c'est pour ça que les gens s'accrochent à quelqu'un qui tout d'un coup vit des choses.

Cela fait penser à la notion de « réalisme émotionnel » développée par Ien Ang pour étudier la réception de Dallas aux Pays-Bas : les spectatrices interrogées jugent réels les sentiments et les émotions qu'elles éprouvent face au feuilleton (Ang, 1989 ; Pasquier, 1999, p. 49). Ici, il s'agit plutôt du réalisme des émotions des personnages. Cependant, on peut aussi lire ce réalisme des émotions dans le processus d'identification qui a lieu pour les auteurs eux-mêmes au moment de l'écriture. La discussion sur la responsabilité des auteurs et de la série ne se pose pas seulement en une discussion sur les réactions possibles du public, la discussion porte aussi sur les propres réactions des auteurs, ce qu'ils feraient à la place des personnages. Ainsi, deux auteurs du séquencier hommes, d'environ 40 ans (soit l'âge du personnage de Benoît, père de Raphaël) réagissent au contenu de la séquence (de Benoît face à son fils au commissariat) de leur propre point de vue. 
Séquenceur 4 : le côté, «Ah tes études » de Benoît, c'est bourrin... C'est plus : « la mort d'un homme, rends-toi compte ».

Séquenceur 1 : Benoît s'en veut de ne pas l'avoir surveillé. Moi, je culpabiliserais un max.

La séquence insiste ensuite sur le sentiment de culpabilité du père, une séquence suivante le montre se posant la question de sa responsabilité. Par ailleurs, la séquence du lendemain montre aussi le père de Benoît prenant la responsabilité du geste à la place de son fils. Ainsi, le sous-titre de cette séquence dans le séquencier est « Benoît pourra-t-il calmer son fils?». C'est ici le père qui est décrit comme accablé d'apprendre que Raphaël risque la prison; de même, la $6^{\mathrm{e}}$ séquence de la journée du mardi s'intitule «Benoît se sent coupable ». Comme pour juger de la vraisemblance de certains détails, les auteurs et acteurs du processus de production se prennent d'abord pour étalon de mesure des réactions du public.

Le rapport à la réalité visé par la série s'appuie donc, à cette étape du développement, sur ces deux régimes d'intrigues, mais aussi sur l'usage d'un ensemble de détails fonctionnant comme effet de réel, en premier lieu ici la mise en scène d'une procédure juridique venant sanctionner cet acte, ainsi que sur une vraisemblance mesurée par la justesse des sentiments des personnages. Par ailleurs, un mécanisme essentiel de la fabrication du feuilleton apparaît ici : les premières personnes qu'il s'agit de convaincre lors de l'écriture, et tout au long du processus de production, ce sont les autres auteurs, les participants du processus de fabrication.

\section{DES HISTOIRES AUX PERSONNAGES}

Cette attention aux personnages et à leurs réactions est plus sensible lors de la deuxième phase de l'écriture, celle de la rédaction des versions dialoguées des scénarios. Une fois le séquencier rédigé par l'atelier séquencier, le directeur d'écriture et un des scénaristes du séquencier nommé pour la semaine « auteur référent » viennent expliquer aux dialoguistes les différentes séquences. Lors de cette réunion, le directeur d'écriture et le référent doivent expliciter leur travail et leurs décisions aux dialoguistes. Les différentes séquences font l'objet de nouvelles discussions qui mettent encore en jeu la cohérence narrative, la vraisemblance et le devenir des personnages. Les dialoguistes reposent ces questions en ayant en tête leurs propres préoccupations professionnelles, qui sont de développer en répliques les séquences décrites, de leur donner un contenu et de donner corps aux personnages. 


\section{Le choix des mots}

Les dialoguistes sont souvent plus attentifs à l'exactitude des termes juridiques à employer. Le geste commis par Raphaël suscite alors à nouveau des discussions sur ses conséquences juridiques à partir des connaissances sur la société que l'on pense partager avec tous, par exemple à partir des faits divers. La crédibilisation passe ainsi par des détails ou des expressions qui fonctionnent comme effet de réel, comme le fait de parler de « tuteur légal » ou encore de dire que Raphaël sera en « liberté surveillée ».

Chef atelier dialogue : est-ce qu'il ne risque pas beaucoup plus parce que c'est prémédité là ?

Dialoguiste 1 : avec cette histoire d'un rugbyman qui avait tué sa femme, ils ont dit que c'était sans préméditation. Même s'il est arrivé avec un flingue, il était bourré.

Dialoguiste 2 : on sait ce qu'il risque mais on reste dans le vague... Quel âge il a ? Directeur d'écriture : il est arrivé à 16 ans.

Chef atelier dialogue $2:$ il est en terminale, c'est un bon élève.

Dialoguiste $3:$ il reste dehors.

Directeur d'écriture : le juge décide de le laisser libre.

Dialoguiste $1:$ tu crois que c'est possible dans la vie?

Dialoguiste 4 : bah non...

Dialoguiste 2 : celui qu'il a planté, c'est un salaud... On peut peut-être parler de « suivi psychiatrique».

Séquenceur 3, auteur référent : oui, « liberté provisoire avant expertise psychiatrique », et il passera devant le juge des enfants.

Ici, on voit que c'est à partir de leurs propres connaissances que les auteurs jugent de la crédibilité des histoires, et qu'une grande attention est portée aux termes employés. Ce sont ces termes qui sont discutés autant que la suite juridique du geste. Le choix des termes justes fonctionne comme une précision de l'effet de réel visé dès l'étape du séquencier par la mention de la procédure juridique. Les termes cités fonctionnent comme des points d'ancrage dans une réalité sociale et pénale. Il s'agit de voir ce dont on peut « parler », de citer pour les dialoguistes des termes clefs à replacer dans les dialogues (comme on le voit dans l'extrait cité plus bas). Il est intéressant de noter que, quand bien même les auteurs s'accordent pour dire que cela ne serait pas possible dans la réalité, la discussion s'engage sur l'utilisation des termes légaux et sur la qualification de la situation juridique. 


\section{Rendre crédible, c'est rendre cohérent}

Ensuite, un des points de préoccupation consiste à développer la séquence en dialogues, en trouvant les arguments et les justifications des uns et des autres La séquence résumée en une phrase le jeudi à 13 heures est devenue tout un paragraphe le lundi matin, lors de la réunion des séquenceurs. Elle doit ensuite se transformer en dialogues une semaine plus tard.

Si la plupart des phrases du séquencier sont déjà au style indirect libre, les développer en répliques demande de connaître les termes exacts ou de nourrir avec des arguments les propos des différents personnages. Les dialogues insistent donc le plus souvent sur les motivations des personnages pour alimenter leurs discours. Ces sujets sont abordés dès l'étape du séquencier. Une partie des préoccupations de dramaturgie et de cohérence amène à produire des justifications et des explications pour tous les actes des personnages : expliquer leur décision aux autres pour les auteurs, que ce soit au niveau du séquencier ou du dialogue, revient le plus souvent à motiver et rendre logique les actions des personnages. C'est un aspect important du processus d'écriture, le public est ici présent dans les esprits, mais le plus souvent de manière implicite : l'important est d'abord d'être compris par ses collègues, et donc aussi à terme par le public. Dans sa version dialoguée définitive, la scène développe ainsi en différents arguments les phrases du séquencier et propose une justification psychologique au geste de Raphaël ainsi qu'un cadre légal à son absence de conséquences juridiques.

\section{Première scène de l'épisode 1777. Dans le bureau de la commissaire Vera Madigan, Benoît est venu chercher Raphaël.}

Benoît et Raphaël sont assis en face du commissaire. Raphaël a l'air absent. Benoît, lui, est accablé. Vera tend un document à Raphaël.

VERA

Signez ici... (Raphaël signe un document). Vous sortez de chez le juge pour enfant?

BENOÎT

Oui... Raphaël est mis en examen.

VERA

Vous avez de la chance qu'il ne parte pas en préventive. Aujourd'hui, ça n'effraye plus personne d'envoyer un mineur en prison. (Véra présente le document à Benoît.) Vous devez signer en tant que représentant légal.

BENOÎT

Le juge a considéré que c'était un geste passionnel. 
Benoît a fini de signer. Véra range le document. Elle s'adresse à Raphaël avec gentillesse.

VERA

Qu'est-ce qui vous a pris ? Vous êtes un garçon qui a reçu une éducation, à qui on a inculqué des valeurs. (Raphaël ne répond pas.) Répondez-moi, que je comprenne...

RAPHAËL

J'aimais Jennifer.

VERA

Vous considérez que l'amour excuse tout?

RAPHAËL

C'est bon. Le juge m'a déjà fait la morale.

(Véra devient plus dure.)

VERA

Ce coup de couteau va vous suivre jusqu'à la fin de votre vie. Il sera marqué dans votre casier judiciaire, vous ne pourrez pas rentrer dans la fonction publique.

RAPHAËL

Je m'en fous.

BENOÎT

Raphaël, change d'attitude, s'il te plaît.

RAPHÄ̈L

Je m'en fous d'avoir une vie de merde. Jennifer est morte, j'ai plus envie de rien...

VERA

Le temps aurait été votre allié... Petit à petit, vous auriez accepté ce décès. Le procès du meurtrier de Jennifer vous aurait aidé à comprendre ce qui s'était passé.

RAPHAËL

C'est pas la peine de vous fatiguer.

VERA

Aujourd'hui, en plus de subir le deuil de votre amie, vous êtes inculpé pour tentative de meurtre. Comment vous pouvez avoir confiance en l'avenir ?

RAPHAËL

C'est quoi l'avenir? Je connais pas ce mot.

VERA

Dans quelques années, vous comprendrez ce qu'il signifie... J'espère qu'il ne sera pas trop tard.

BENOÎT

On peut y aller? 


\section{Un temps.}

VERA

Oui...

Benoît se lève. Pas Raphaël.

BENOÎT

Tu viens?

Raphaël se lève mollement.

VERA

Si vous avez besoin de quoi que ce soit, vous pouvez venir me voir.

BENOÎT

Merci...

Véra regarde tristement partir Benoît et Raphaël ${ }^{8}$.

Des réflexions sur les mineurs en prison se retrouvent dans le dialogue ainsi qu'un ensemble de termes juridiques qui viennent poser un cadre légal au geste. Cela se retrouve dans la péripétie qui nous occupe avec le souci de donner les conséquences du geste du jeune homme pour le reste de sa vie, la commissaire évoquant dans l'échange l'impossibilité pour le fils de trouver un emploi dans la fonction publique, ou encore sa difficulté à se reconstruire après un tel geste.

Les dialogues insistent sur les raisons de l'acte, le geste est mis en intrigue cohérente avec des motifs, des raisons. C'est un des ressorts de la construction des dialogues.

Chef atelier dialogue : Les situations sont extrêmement complexes mais il faut les rendre simples. Il faut les rendre appréhendables. La situation complexe, c'est la situation où les gens se demandent : mais pourquoi ce mec-là fait ça ? C'est que les gens ne comprennent pas pourquoi il le fait. Alors, à partir du moment où le téléspectateur ne comprend pas pourquoi un personnage fait ça, on est mort. C'est-à-dire que, tout d'un coup, il croit plus en le personnage, il croit plus en l'histoire, il croit plus en rien. Donc, c'est de rendre cette situation appréhendable

La construction de la cohérence dramaturgique des personnages est un élément important de la construction de la vraisemblance par la série, qui passe, dans l'exemple étudié, par la mise en avant de la justification psychologique

8. Extrait de l'épisode 1177. 
de la tentative de meurtre par la passion amoureuse. Les dialoguistes portent un autre regard sur les personnages, une partie de leur travail demande de comprendre les raisons d'agir des différents personnages pour pouvoir rendre leurs sentiments et leurs motivations en paroles. Les dialogues sont un élément essentiel de l'économie narrative du feuilleton, les personnages passent beaucoup de temps à expliciter leurs motifs et à discuter de leurs actions avec les autres. Ainsi, c'est à travers les dialogues qu'est développée plus haut la justification de l'acte de Raphaël, ce sont les confidences échangées lors des séquences insistant sur le deuil de l'adolescent qui proposent une justification de son geste, et une condamnation de celui-ci par son père.

Lors de cette étape des dialogues, un des mécanismes de rapport au contenu de la série est le plus perceptible, c'est-à-dire la recherche de motivations et de justifications pour les actions de chacun des personnages. Cette recherche de cohérence passe par de très nombreuses discussions, et c'est elle qui permet d'emporter l'accord. Ici, il faut viser l'accord minimal des différents participants sur la cohérence des actions des personnages, et ceci en leur fournissant des motifs, des bonnes raisons d'agir.

L'étape des dialogues permet d'analyser comment le rapport à la réalité de la série est construit par son mécanisme même d'écriture, qui est aussi un processus d'argumentation et d'explicitation des actions des personnages, elle rend aussi plus précise la visée de l'effet de réel par la série.

\section{LES DESSOUS TECHNIQUES DE LA VRAISEMBLANCE}

Les discussions sur la vraisemblance de la série n'ont pas lieu seulement au niveau de l'écriture mais aussi au moment du tournage et de sa préparation. Le tournage quotidien s'accommode ainsi, à son niveau, des invraisemblances et des rebondissements de la série en les retraduisant en contraintes techniques selon la limite des moyens de production engagés. Comme Dominique Pasquier l'avait décrit à propos du tournage d'Hélène et les garçons (Pasquier, 2008), ou Philippe Le Guern à propos de Julie Lescaut (Le Guern, 2002), les tournages de télévision sont très intensifs et rapides. Le feuilleton est diffusé toute l'année à raison de 260 épisodes par an. Pour tenir cette exigence, l'organisation du tournage est très serrée : chaque semaine d'épisodes est tournée en environ une semaine de travail. Pour gagner du temps, l'ordre des séquences au tournage ne dépend pas de l'ordre chronologique de l'action, mais de l'organisation du plan de tournage du réalisateur. Le temps de tournage est 
très minuté, 67 séquences d'intérieurs sont tournées en 5 jours. On est dans une organisation presque industrielle de ce monde de l'art avec une division du travail extrêmement poussée.

\section{L'attention aux détails}

Cette attention aux détails spécifique aux contraintes du tournage se manifeste dès les premières étapes de l'écriture. Le séquencier est relu par la chaîne et par les responsables du tournage à Marseille. Chaque semaine, le directeur artistique, le directeur d'acteur, le responsable du planning, les responsables de la production de France 3 et de la maison de production, et une coordinatrice d'écriture émettent des remarques sur le séquencier ( « les remarques du plateau »). Celles-ci portent principalement sur la faisabilité des scènes, sur les possibilités du tournage, mais elles contiennent aussi des réflexions sur la vraisemblance et la cohérence des intrigues. Et ici, en particulier sur les conséquences juridiques de cette agression : «Si Raphaël plante réellement Frémont dans le ventre, il encourt de graves poursuites, largement au-delà de la liberté jusqu'à l'audition. Même si Frémont ne porte pas plainte et même si Raphaël est mineur, dans la mesure où il a gravement blessé Frémont, l'État se portera au minimum partie civile. Raphaël sera déféré devant un juge pour enfants, etc. ${ }^{9}$. " Il semble qu'au fur et à mesure du processus de production, l'effet de réel est visé de manière de plus en plus concrète, ici encore au travers des termes juridiques mais aussi par la nature et l'emplacement de la blessure. De même, quelques semaines plus tard, lors d'une réunion de préparation du tournage ${ }^{10}$, l'assistant du directeur artistique qui donne des indications sur les accessoires, les costumes et la mise en scène des différentes séquences, souligne l'invraisemblance de la péripétie et de ses conséquences.

L'assistant du directeur artistique a les scénarios devant les yeux et commente les séquences en donnant des indications sur les accessoires, ou encore la mise en scène de la séquence. Il commente la séquence 1777/01 (première séquence de l'épisode diffusé le lundi, soit le lendemain du geste). Cette séquence dont la version dialoguée a été reproduite plus haut montre le père de Raphaël, Benoît, venant le chercher au commissariat.

9. Extrait des « Remarques du plateau » concernant les épisodes en question, distribuées lors de la réunion dialogues.

10. Réunion « mise en scène et accessoires » qui réunit l'assistant du directeur artistique, le réalisateur, le second assistant réalisation, le responsable des accessoires, le responsable des décors. 
Assistant directeur artistique : «Le document» (le scénario ne précise pas de quel document il s'agit), c'est quoi ce document? Nous, on va faire que Benoît signe (le scénario indique que c'est Raphaël qui signe un document juridique) et Raphaël ne signe rien. (Au responsable des accessoires) T'appelles le commissariat. Que ce soit en deux exemplaires. Raphaël n'aura pas de menottes.

Les responsables du tournage mettent un soin particulier à mettre en scène, à jouer avec les vrais documents. L'usage du véritable document qu'on verra à peine à l'écran fonctionne presque comme effet de réel pour les fabricants eux-mêmes. Il semble lors de cette réunion évident à tous les participants qu'il faut montrer le vrai document. Par ailleurs, la volonté de cohérence se retrouve au moment du tournage dans l'attention à des détails signifiants pour la narration, et la vraisemblance de la part des différents protagonistes. Ainsi, lors de cette même journée de tournage est tournée la scène où Benoît et son fils reviennent chez eux après la nuit passée au commissariat. Lors de la préparation, la semaine précédant le tournage, l'assistant du directeur artistique a ainsi précisé aux assistants du réalisateur qui transmettent ensuite l'information au maquillage et à la coiffure, ainsi qu' aux costumes, que les deux personnages doivent avoir l'air d'avoir passé la nuit au poste. La feuille de service distribuée la veille du tournage précise donc pour cette scène : "Séquence 11776/6 : Raphaël et Benoît raccord de la veille. Tâche de sang sur la manche de Raphaël ». Au moment du tournage, la coiffeuse qui surveille la scène derrière l'épaule du réalisateur vient décoiffer l'acteur qui joue Raphaël.

Tout un ensemble d'actions viennent ainsi replacer les scènes dans le quotidien : le commissariat est traversé par de nombreuses personnes, lors de la scène, on voit en fond des figurants en policiers ou en prévenus traversant la pièce. De même, dans de très nombreuses scènes, pour compenser l'omniprésence des dialogues, les personnages accomplissent des actions diverses qui les replacent dans la vie quotidienne et pratique. Ainsi, lorsque Nathan vient consoler son ami Raphaël, sur les indications de la direction artistique, la note de service ${ }^{11}$ détaille comment cette scène de confidence est replacée dans le cadre de la vie d'adolescents actuels :

Séquence 1776/14 : Raphaël regarde la vidéo de Jennifer sur son ordinateur portable. Prévoir écouteurs. Paquet de chips consommable et renouvelable + sac de cours de Nathan.

11. La feuille de service est une feuille de format A4 distribuée à tous la veille du tournage et qui récapitule l'ordre des séquences tournées, les horaires d'arrivée des différents professionnels, ainsi que les recommandations en matière d'accessoires, de costumes et de maquillage. 
La présence du sac de cours de Nathan vient aussi mettre en lumière le fait que Raphaël a passé la journée chez lui, des détails sont insérés dans le décor qui replacent la scène dans un univers commun de représentation, et replace l'extraordinaire dans l'ordinaire de la vie de tous les jours.

\section{Ce que l'on montre et ce que l'on cache : quand le narratif devient contrainte technique}

Le point de vue du plateau retraduit en questions pratiques ce dilemme moral et retranscrit les questions de vraisemblance en une question de mise en scène et de jeu avec ce qui est montré. Les nécessités techniques et les contraintes se mêlent à la revendication morale. La question de la blessure et de sa gravité, ainsi que de la mise en scène de l'acte posent tout un ensemble de questions pratiques et techniques lors de la préparation du tournage et du tournage proprement dit. Lors de la réunion de mise en scène :

Réunion mise en scène, mardi 3 février :

Assistant directeur artistique : La 76/16, intérieur commissariat. Raphaël est déjà en place, sur les sièges d'attente, Vera signe la décharge, le transfert. Raphaël arrive avec les mains dans les poches. Au fait, il est droitier ou gaucher ? Elle serait de quel côté la blessure... bref vous vérifiez ça. Donc Raph pousse Samia, on ne fait pas d'insert ${ }^{12}$ sur la blessure, on voit le couteau quand il fonce sur Frémont mais pas le couteau qui entre dans la chair. On cliffe ${ }^{13}$ sur les deux corps emboîtés.

Réalisateur : Je voudrais aller le plus loin possible dans le mouvement.

Assistant directeur artistique : Moi, je veux pas d'installation avec une poche de sang, ça veut dire changer de costume pour Frémont.

Réalisateur : Pour qu'on voie bien, il ne faut pas qu'ils soient tous les deux en sombre. (Il souligne la question des menottes et de l'utilisation d'un couteau rétractable pour les accessoires.)

Assistant directeur artistique : Il faut prévoir une protection dorsale pour l'acteur pour protéger les abdos, que Raphaël puisse y aller gaiement et on fera un casting de couteaux rétractables.

12. Un insert désigne le fait de faire un plan particulier sur un détail, ici, cela veut dire qu'il ne veut pas qu'on fasse un plan sur la blessure.

13. «Cliffer » est le terme utilisé par les fabricants de Plus belle la vie pour désigner la manière de filmer les derniers plans de la dernière séquence, cela vient du « cliff », diminutif de cliffhanger. Ici, les cliffs sont filmés soit avec un zoom sur les visages des personnages, soit en « décliffant », c'est-à-dire en élargissant le plan pour prendre en compte toute la scène. 
La scène pose alors en des questions pratiques et techniques les questions de vraisemblance : la nécessité d'avoir un couteau rétractable, une poche de sang, la manière de filmer, les déplacements des acteurs. Il s'agit de rendre crédible que Raphaël attaque Frémont en plein commissariat, mais aussi que ce geste ne soit pas trop montré : il est donc précisé que la blessure ne sera pas filmée, cela évite d'avoir à en faire une au maquillage, et cela permet aussi de ne pas insister sur cet aspect. De la même manière, on ne verra pas la victime saigner, pour des raisons à la fois techniques inscrites dans l'économie du tournage (ne pas gâcher un costume en le tâchant de sang, et des problèmes de raccord : tourner la scène plusieurs fois amène à tâcher plusieurs costumes), mais cela permet dans le même temps de ne pas insister sur la gravité de la blessure.

La scène s'achève dans le scénario sur Frémont qui s'effondre et la réalisation amplifie cet aspect. Lors la scène d'agression, sur les indications de la direction artistique, deux caméras sont dirigées vers Frémont, une seule vers Raphaël, le geste est filmé de dos, les trois caméras ${ }^{14}$ finissent sur le visage de Frémont. On insiste aussi à la fois sur la méchanceté de Frémont et sur la noirceur manipulatrice du personnage de Kamsky, ce qui a pour effet de relativiser le geste de Raphaël. L'acteur qui joue le personnage de Charles Frémont insiste sur le côté cabot et goguenard du personnage : celui-ci, qui traverse menotté le commissariat entouré de deux policiers, les nargue et se moque d'eux, juste avant d'être poignardé. Le comédien rajoute des petites phrases pour montrer son arrogance, et se tord la bouche quand il se fait poignarder. Il est montré dans des séquences de la semaine suivante paradant à l'hôpital, méprisant les policiers.

On voit que la question de la vraisemblance, posée au départ à travers l'histoire et le contenu même de la séquence, se repose ensuite à tous les niveaux de la fabrication, se traduisant selon les étapes dans les termes des différents intervenants. Ainsi, pour faire la chambre d'hôpital et garder la continuité des séquences, l'accessoiriste doit faire attention à ce que les cigares et le roman apporté par la fille de Frémont à l'hôpital apparaissent bien dans les scènes suivantes pourtant situées avant dans l'ordre de tournage. Les cigares viennent signaler l'aisance et l'appartenance sociale de Frémont, tout en dénotant son caractère de «méchant ». Il s'agit donc aussi, en ne montrant pas la blessure, de faciliter les raccords. C'est donc pour des raisons d'organisation, de même que pour ne pas insister sur la gravité du geste, que la blessure n'est jamais

14. Le tournage en décor utilise en effet trois caméras pour filmer directement les actions et réactions des personnages ainsi que les plans larges. 
montrée à l'écran. Les préoccupations de vraisemblance mêlent à la fois des considérations techniques et une attention aux réactions du public.

Par souci de vraisemblance, une attention est accordée à tout un ensemble de détails qui viennent replacer les rebondissements incroyables de la série dans le cadre de la vie quotidienne tout en les traduisant dans l'économie du tournage de la série... Même si elle met en scène de nombreuses intrigues policières et séquences d'action, les fabricants de la série ont été amenés à trouver, en fonction de leur économie de production, leur manière singulière de représenter la réalité sociale.

\section{RAPPORT À LA RÉALITÉ SOCIALE ET REPRÉSENTATION DU PUBLIC ET DE LA RÉCEPTION}

La gestion de cette contradiction qui pose le réalisme comme un critère de décision et une référence tout au long du processus de production, et la façon dont elle se pose aux différentes étapes de la fabrication, sont sous-tendues par les représentations du public et de la manière de regarder la télévision des différents acteurs. Cela est particulièrement perceptible à travers ce geste puisqu'il pose pour les fabricants le problème de leur responsabilité vis-à-vis du public. À cette occasion, on peut voir comment la préoccupation du public se pose différemment selon la place des uns et des autres dans le processus de production. Enfin, les débats décrits du séquencier au tournage mettent en œuvre une certaine conception de l'adhésion à la fiction, et par là une certaine représentation du public.

\section{Sens des responsabilités : croire aux effets des médias}

Ce geste et ses conséquences soulèvent d'autres types de réflexion qui mêlent au souci de vraisemblance minimale une conscience de leur responsabilité morale par les fabricants du feuilleton. Le fait de laisser la tentative de meurtre de cet adolescent sans conséquences légales soulève aussi des problèmes moraux chez les auteurs. Il s'agit de souligner la gravité du geste. L'attention au public et à ses réactions est également présente lorsque les auteurs réfléchissent au message, à la morale délivrée par l'intrigue. Comme l'observait Sabine Chalvon-Demersay à propos des adaptations télévisées, les considérations sur la cohérence des personnages sont souvent liées à des réflexions sur leur comportement moral (Chalvon- Demersay, 2007a). Si la vraisemblance 
est évoquée, c'est aussi un sentiment de responsabilité vis-à-vis du public et de ce qui est raconté dans la série qui apparaît. Un des scénaristes évoque ce sentiment de responsabilité :

Séquenceur 4 : En tout cas, il y a une grande responsabilité. Je pense que tout le monde a conscience que... on dit quelque chose sur les sujets qu'on aborde. Très clairement, on dit quelque chose, et donc on se pose la question de qu'estce que ça raconte? Quelqu'un qui regarde ça, qu'est-ce qu'il se dit?

Ce sentiment de responsabilité porte en creux une conception du public et de sa réception. Le succès de la série ainsi que son réalisme affiché incitent les fabricants à craindre que les spectateurs, ou certains d'entre eux, entrent pleinement dans l'effet de croyance mis en place par le réalisme de la série. On voit ici percer en partie l'ambition " pédagogique » des auteurs et de la série, parfois de manière très explicite. Car celle-ci permet de faire passer des messages, le fait d'être diffusé sur une chaîne publique est alors essentiel à la définition de la série, comme l'explique le directeur artistique : "Comme on a quand même vraiment une mission de service public, qu'on essaie de tenir, si tu veux. Alerter les gens sur un certain nombre de phénomènes de société, essayer de faire avancer certaines causes ». C'est aussi cette volonté de faire passer des informations qui est soulignée par le producteur dans l'interview citée plus haut: "On est tout à fait à même de passer des messages, se félicite Hubert Besson. Par exemple, il y a deux ans, nous avons traité du baptême citoyen. Nous avons aussi parlé du don du sang. Ce genre d'histoire B a un impact sur le comportement des téléspectateurs. Cependant, nous devons faire ça avec beaucoup de modération, avec un grand sens civique, parce qu'on sait qu'il y aura des répercussions sur nos téléspectateurs ${ }^{15}$. » Cette représentation d'une mission est liée au réalisme de la série, puisque c'est parce que le feuilleton passe pour réaliste qu'il peut être l'occasion de transmettre des messages, ou d'alerter le public sur un problème. Cela tend à donner l'image d'un public entrant pleinement dans l'effet de réel et adhérant complètement au monde de la série. Cela semble montrer que ces professionnels des médias partagent l'idée courante d'un effet des médias sur leur public, et qu'ils adaptent leur travail à cette croyance. Cette représentation des effets de la télévision rejoint en partie une des premières manières de penser l'influence de la télévision (Maigret, 2003, p. 59) et repose en partie sur une conception du public de la télévision comme «passif» (Le Grignou, 2003, p. 16) ${ }^{16}$.

15. Cité dans Slate.fr, par Pierre Langlais, 7 février 2010.

16. Cette conception du public de la télévision était déjà soulignée par Pierre Bourdieu et JeanClaude Passeron (Bourdieu \& Passeron, 1963). 
Cette attention est présente en filigrane dans les discussions entre auteurs, derrière les questions de vraisemblance. En effet, les scénaristes développent une réticence à parler du public, comme l'a souligné Dominique Pasquier dans Les scénaristes de télévision (1995, p. 95) : la manière de réagir du public n'est pas souvent mentionnée explicitement dans les discussions, mais elle dessine en creux tout un ensemble de limites de ce qu'il est possible de dire ou non. Dans le processus de fabrication, la référence aux réactions des téléspectateurs est portée différemment selon les intervenants. Ainsi, tout au long de la chaîne de fabrication une concrétisation de l'attention à l'effet de réel est perceptible : la mention d'une procédure juridique devient une recherche des termes exacts, puis la quête du document authentique à montrer à l'écran. Cela se retrouve plus particulièrement dans la manière de parler du public : cette préoccupation est principalement portée par le diffuseur et le producteur. Lors de la réunion dialogue, le directeur d'écriture mentionne que le producteur n'est pas enthousiasmé par ce développement de l'intrigue. La responsable du feuilleton au sein de la chaîne, qui fait des remarques chaque semaine sur les séquenciers, déplore par ailleurs l'usage de la violence par des adolescents mis en scène dans la série (quelques jours plus tard, c'est une autre adolescente qui vole une arme à feu pour protéger son père) comme un mauvais exemple à donner à l'antenne et une dérive de la part des auteurs. Dans la répartition des compétences, ce sont essentiellement le producteur et la chaîne de diffusion qui portent le plus directement la préoccupation des réactions possibles du public, car ils sont aussi les porteurs des préoccupations de rentabilité et de succès économique du produit.

\section{Penser les personnages, penser la réception}

L'attention à la cohérence des personnages, à la justification de leurs actes dessine aussi, comme on l'a vu, une manière implicite de concevoir la réception du feuilleton par les téléspectateurs. En effet, les personnages et leurs actions sont définis et pensés à partir de leurs bonnes raisons d'agir. Tout le processus d'écriture est constitué de débats et d'argumentations qui cherchent à expliciter les motivations des individus. Il s'agit donc de rendre intelligible aux autres auteurs les comportements des personnages et par là les décisions narratives en exhibant leur rationalité. Le critère de ce qui sera compréhensible et accepté par tous, testé à travers tout le processus d'écriture, devient aussi le modèle de la réception du feuilleton par les spectateurs, et de l'adhésion à la fiction : le spectateur y croira car il comprendra pourquoi les personnages agissent ainsi. La manière dont les représentations du public et de 
la réception travaillent la mise au point des exigences narratives de la série ressort bien des propos d'un responsable de l'atelier dialogue (comme dans ceux cités plus haut) :

Chef atelier dialogue : Et à un moment donné, pour chaque scène, il faut trouver une couleur. Qu'est-ce que le personnage va vivre dans cette scène-là ? Et pousser cette situation. Le directeur d'écriture met en place des situations où les gens sont dans des situations très, très, très complexes et, nous, notre travail, c'est que cette situation soit le plus en exergue possible... Mais que ça soit de façon, d'une façon simple, c'est-à-dire que faut pas rentrer dans des sentiments extrêmement complexes où on ne comprend rien. Non, il faut que ça soit simple.

La compréhension, l'adhésion au feuilleton passe alors par la simplicité et l'unicité des motivations des personnages. En effet, souvent, les auteurs dès le séquencier essaient d'éviter les scènes contenant une double intention : dans une séquence, on ne doit suivre en principe qu'une seule action à la fois. De la même manière, les responsables des dialogues ont comme règle de ne pas faire de second degré dans les intrigues, et en particulier de ne pas faire de comédie, de répliques drôles dans les séquences relevant de l'intrigue policière. L'unicité et la simplicité des personnages doivent être le support de l'adhésion d'un public nombreux et divers.

\section{Trouver l'accord entre soi pour correspondre au public}

Le mécanisme de recherche d'un accord sur les motivations des actions et sur les détails du scénario semble fonctionner en partie comme une négociation portant sur les catégories de classement et de perception du réel des uns et des autres : en cherchant l'accord avec les autres intervenants du processus de production, c'est un accord avec les catégories de perception du public qui est visé. Cette fonction des lectures multiples des scénarios est mise en évidence par Sabine Chalvon-Demersay et Dominique Pasquier : "Tout se passe comme si les réactions des premiers lecteurs constituaient un modèle réduit des réactions du public » (Chalvon-Demersay \& Pasquier, 1991, pp. 106-107).

Le partage de catégories communes de perception du monde social est ainsi visé et mis en œuvre tout au long du processus de production, dans une recherche du "plus grand dénominateur commun », tel que M. Baxandall l'a décrit à propos des peintres travaillant sur commande au XVe siècle (Baxandall, 1985, p. 64). 
Cela amène à entrer dans le détail de ce qui se joue dans le phénomène de croyance et d'adhésion au monde de la fiction réaliste, tel qu'il est décrit par exemple par Bourdieu à propos de l'œuvre de Flaubert : il relie en effet l'effet de réel de la fiction réaliste aux partages de structures mentales par le lecteur et l'auteur (Bourdieu, 2000, pp. 457-458) en décrivant ainsi le mode de fonctionnement de "cette forme très particulière de croyance ", qui repose, selon lui, "sur l'accord entre les présupposés qu'il engage et ceux que nous engageons dans l'expérience ordinaire du monde » (Bourdieu, 2000, p. 60). Or, lors de la fabrication de la série, les différents intervenants utilisent leur propre expérience ordinaire du monde pour tester et éprouver le fonctionnement des « effets de réel », le réalisme de la série. Les différentes discussions reproduites ici démontrent ce fonctionnement : c'est à chaque fois à partir de leur propre jugement et perception du monde social que les différents intervenants évaluent la pertinence, la vraisemblance des intrigues. Par exemple, lors de la préparation du tournage d'une scène d'hôpital montrant Frémont blessé, la discussion porte sur le fait de savoir quel type d'accompagnement médical demande la blessure, quel type de nourriture. Comme sur le plan juridique évoqué plus haut, ce sont les connaissances de chacun qui construisent la scène et les décisions prises :

Assistant directeur artistique : On ne met pas de machine à bip ridicule. Il ne peut pas manger, il est sous morphine, on lui met un cathéter.

Réalisateur : C'est pas grave, c'est juste un cathéter, on met une perf pour Frémont.

Assistant directeur artistique : Il est hors de question que Frémont ait une plaquette de beurre, il a une compote de pomme et c'est tout. Léo donne un coup dans la compote qui vole en éclats.

On retrouve ces ressorts dès l'écriture : ce qu'il faut atteindre, c'est un accord minimal avec les catégories de perception et les connaissances légales ou médicales les plus communes, jugées selon celles des fabricants de la série, les représentations que l'on peut avoir des papiers à signer dans un commissariat ou de la nourriture donnée à l'hôpital. Dans ces discussions, qui ont lieu de manière presque identique à chaque étape, chacun se fait expert du système juridique ou des termes médicaux. À partir de leurs propres connaissances et de leur propre expérience sociale, les auteurs jugent, avec leur propre sens commun, de ce qui serait possible. C'est donc en fonction de leur propre expérience mais aussi pour correspondre à l'expérience sociale supposée des téléspectateurs que sont ajoutés ou spécifiés des détails juridiques par exemple. Cette manière d'écrire est décrite par Sabine Chalvon-Demersay dans son analyse des scéna- 
rios d'amateurs : «L'auteur utilise des personnages, des objets, des contextes, des intrigues qui sont mis à sa disposition par la culture environnante et par les conventions littéraires disponibles. Il puise dans un répertoire commun les références qui lui plaisent et qui lui sont accessibles, les combine, les organise pour fabriquer son texte à lui » (Chalvon-Demersay, 1994, p. 13). Le travail d'écriture et de fabrication de la série pioche dans un ensemble de références considérées comme communes à tous par les scénaristes, mais aussi par les intervenants leur succédant dans la chaîne de production. Cette perspective mériterait cependant d'être approfondie et nuancée : les conceptions du public ou les définitions de ce qui est vraisemblable par les fabricants peuvent différer en raison de leur place dans le processus de production (Pasquier, 1995, p. 6), comme on l'a vu, mais aussi en fonction de leurs trajectoires, de leurs socialisations différenciées, de leurs références culturelles.

\section{Conclusion}

Le processus de fabrication de ce feuilleton télévisé est donc traversé par une contradiction entre le caractère invraisemblable de ses intrigues et l'ambition de réalisme inscrite dans la définition originelle de la série. Ce paradoxe se traduit par un souci de réalisme qui se décline en un ensemble de questions dramaturgiques et techniques, en enjeux professionnels tout au long de la chaîne de coopération. L'attention au réalisme devient par là une convention de travail qui coordonne les différentes étapes à travers ce même critère de jugement. Si les acteurs du processus de production travaillent avec une idée des connaissances partagées par le public le plus large, des codes à employer, des niveaux de langue, des indices que peut déchiffrer un téléspectateur pour comprendre ce qui se déroule dans la série, cette idée est largement implicite, et ils prennent le plus souvent eux-mêmes et leurs collègues comme premier public sur qui tester la vraisemblance. La production de la série consiste alors aussi en une manière particulière d'interpréter le sens de celle-ci par ses fabricants, à travers la recherche d'un accord sur les catégories de perception du monde social des uns et des autres, comme une première expérimentation sur un public. Les discussions qui émaillent la fabrication de la série tendent à reconstruire en particulier la volonté de réalisme en une attention à la cohérence et à la motivation des actions des personnages. C'est parce qu'il est une chaîne d'argumentation devant convaincre les autres intervenants que le processus d'écriture tend à accompagner la recherche d'effets de réel sur des détails précis qui encadrent l'action d'une attention à la cohérence. Cette manière de concevoir ce qui amènera l'adhésion de tous à ce qui est raconté 
est par là sous-tendue par les représentations du public et de la manière de regarder la télévision portées par ces professionnels, qui travaillent à partir de leurs connaissances propres et de leurs propres réactions. La fabrication du feuilleton est ainsi travaillée par une contradiction inhérente à la définition même de la série. Celle-ci se donne pour cadre la vie quotidienne d'un quartier populaire de Marseille tout en intégrant des rebondissements haletants dans sa narration. Les fabricants de la série sont alors confrontés à la mission de rendre crédible l'incroyable, exigence qui repose sur leurs propres conceptions de l'adhésion à la fiction, qui semble être fondée ici sur le principe d'une identification et d'une compréhension des actions des personnages par les téléspectateurs. Ainsi, la représentation de ce qui est vraisemblable pour une fiction télévisée est aussi liée à leurs représentations de la manière de regarder la télévision. Celles-ci pourraient être reliées à leurs pratiques culturelles, à leur rapport à la culture, ainsi qu'à leurs trajectoires professionnelles, car leur travail à la télévision pose pour ces différents acteurs la question de leur rapport à la culture populaire. 


\section{RÉFÉRENCES}

ANG I. (1989), Watching Dallas. Soap opera and the melodramatic imagination, Londres, Routledge.

BARTHES R. (1968), « L’effet de réel », Communications, n 11, pp. 84-89.

BAXANDALL M. (1985), L'œil du Quattrocento. L'usage de la peinture dans l'Italie de la Renaissance, Paris, Gallimard.

BECKER H.S. (1988), Les mondes de l'art, Paris, Flammarion.

BOURDIEU P. (2000), Les règles de l'art. Genèse et structure du champ littéraire, Paris, Seuil.

BOURDIEU P. \& PASSERON J-C (1963), « Sociologue des mythologies et mythologies des sociologues », Les Temps modernes, $\mathrm{n}^{\circ}$ 211, pp. 998-1021.

CANTOR M.G. \& PINGREE S. (1983), The soap opera, Beverly Hills, Sage Publications.

CHALVON-DEMERSAY S. (2007a), « Des personnages de si près tenus », Qualitative Sociology Review, Vol. III, Issue 3, December 2007.

CHALVON-DEMERSAY S. (2007b), « Les scrupules de Robinson : les professionnels face à leurs responsabilités morales », Médiamoporhoses, numéro hors-série.

CHALVON-DEMERSAY S. (1994), Mille scénarios. Une enquête sur l'imagination en temps de crise. Paris, Métaillé, Paris, coll. Leçons de choses.

CHALVON-DEMERSAY S. \& PASQUIER D. (1991), « La naissance d'un feuilleton français », Réseaux, hors série, pp. 99-116.

ECO U. (1993), De superman au surhomme, Paris, Grasset.

LE GRIGNOU B. (2003), Du côté du public, Paris, Economica.

LE GUERN, P. (2002), « Les professionnels de la profession : une enquête sur le tournage de la série Julie Lescaut », dans BEYLOT P. \& SELLIER G. (Éds), Les séries policières, Paris, L'Harmattan-Ina.

MAIGRET E. (2003), Sociologie de la communication et des médias, Paris, Armand Colin.

PASQUIER D. (2008), « Conflits professionnels et luttes pour la visibilité à la télévision française », Ethnologie française, 38.

PASQUIER D. (1999), La culture des sentiments. L'expérience télévisuelle des adolescents. Paris, Éditions Maison des Sciences de l'homme.

PASQUIER D. (1995), Les scénaristes de télévision, Paris, Nathan.

PASSERON J.-C. (1991), « L'illusion romanesque », Le raisonnement sociologique, Paris, Nathan, pp. 207-225. 